CAmerican Psychological Association, 2022. This paper is not the copy of record and may not exactly replicate the authoritative document published in the APA journal.

The final article is available at https://doi.org/10.1037/emo0001111.

\title{
Collective Emotions during the COVID-19 Outbreak
}

\author{
Hannah Metzler 1,2,3,4,5, Bernard Rimé ${ }^{6}$, Max Pellert ${ }^{1,2,5}$, Thomas \\ Niederkrotenthaler ${ }^{3}$, Anna Di Natale ${ }^{1,2,5}$ and David Garcia ${ }^{1,2,5}$ \\ ${ }^{1}$ Complexity Science Hub Vienna, Josefstaedter Strasse 391080 Vienna, Austria \\ ${ }^{2}$ Center for Medical Statistics, Informatics and Intelligent Systems, Medical University of Vienna, \\ Spitalgasse 23, 1090 Vienna, Austria \\ ${ }^{3}$ Unit Suicide Research \& Mental Health Promotion, Department of Social and Preventive \\ Medicine, Center for Public Health, Medical University of Vienna, Kinderspitalgasse 15, 1090 \\ Vienna, Austria \\ ${ }^{4}$ Institute for Globally Distributed Open Research and Education, Austria \\ ${ }^{5}$ Institute of Interactive Systems and Data Science, Faculty of Computer Science and \\ Biomedical Engineering, Graz University of Technology, Inffeldgasse 16C, 8010 Graz, Austria \\ ${ }^{6}$ Psychological Sciences Research Institute, University of Louvain, Place du Cardinal Mercier, \\ 10 bte L3.05.01, 1348 Louvain-la-Neuve, Belgium
}

Hannah Metzler https://orcid.org/0000-0001-9254-3675

Bernard Rimé https://orcid.org/0000-0001-8487-575X

Max Pellert https://orcid.org/0000-0002-6557-7607

Thomas Niederkrotenthaler https://orcid.org/0000-0001-9550-628X

Anna Di Natale https://orcid.org/0000-0002-0592-0274

David Garcia https://orcid.org/0000-0002-2820-9151

Data availability statement: All code and data are available at https://osf.io/736kc/.

Conflict of interest: The authors have no conflict of interest to report.

Correspondence concerning this article should be addressed to Hannah Metzler, Complexity Science Hub Vienna, Josefstaedter Strasse 391080 Vienna, Austria. Email: metzler@csh.ac.at. 


\begin{abstract}
The COVID-19 pandemic has exposed the world's population to unprecedented health threats and changes to social life. High uncertainty about the novel disease and its social and economic consequences, together with increasingly stringent governmental measures against the spread of the virus, likely elicited strong emotional responses. We analyzed the digital traces of emotional expressions in tweets during five weeks after the start of outbreaks in 18 countries and six different languages. We observed an early strong upsurge of anxiety-related terms in all countries, which was related to the growth in cases and increases in the stringency of governmental measures. Anxiety expression gradually relaxed once stringent measures were in place, possibly indicating that people were reassured. Sadness terms rose and anger terms decreased with or after an increase in the stringency of measures and remained stable as long as measures were in place. Positive emotion words only decreased slightly and briefly in a few countries. Our results reveal some of the most enduring changes in emotional expression observed in long periods of social media data. Such sustained emotional expression could indicate that interactions between users led to the emergence of collective emotions. Words that frequently occurred in tweets suggest a shift in topics of conversation across all emotions, from political ones in 2019, to pandemic related issues during the outbreak, including everyday life changes, other people, and health. This kind of time-sensitive analyses of large-scale samples of emotional expression have the potential to inform risk communication.
\end{abstract}

Keywords: collective emotions, pandemic, COVID-19, social media, psycholinguistics 


\section{Introduction}

The COVID-19 pandemic has exposed people from all over the world to unexpected and unprecedented health threats and drastic changes to their social lives. High uncertainty about the novel disease and its social and economic consequences, together with increasingly stringent governmental measures against the spread of the virus, likely elicited strong emotional responses in many people. When people experience strong emotions, they tend to share these experiences with others (Rimé, 2009). As they communicate with each other, they do not only exchange information about recent events, but also influence each other's emotional states (Rimé, 2009). When large groups of people emotionally respond to the same situation while interacting with each other, their interaction can lead to the emergence of collective emotions (Durkheim, 1912), which involve changes in the quality, magnitude and duration of the emotional experience (Goldenberg et al., 2020). Automated text analysis of daily social media records allows capturing expressions of emotion in natural online conversations between people (Garcia \& Rimé, 2019). In addition, social media are a particularly interesting data source during the early COVID-19 outbreak, as people's social lives moved online to a larger extent than ever before.

The present study used Twitter data from 18 countries around the world during the first five weeks of the COVID-19 outbreak to observe the evolution of four basic emotional states: anxiety, sadness, anger and positive emotions. These emotions are associated with crisis responses (see Kim \& Niedereppe, 2013), and highly relevant to behavior during a pandemic (Karademas et al., 2013). Importantly, they can also be traced using existing and validated automated text analysis methods (Garcia et al., 2021; Pellert et al., 2021). Our methodological approach builds on studies of emotional responses on social media to one-off catastrophic events, like terrorist attacks (Garcia \& Rimé, 2019), false missile alerts (N. M. Jones \& Silver, 2020), school shootings (Doré et al., 2015; N. M. Jones et al., 2016), campus lockdowns (N. M. Jones et al., 2017) and natural disasters (Gruebner et al., 2017). Yet, in contrast to these events, the pandemic poses a continuous threat evolving over time, presents a global crisis of unprecedented magnitude, and affects all social media users personally. As people used social media during the COVID-19 outbreak, they may have looked for information, shared information and feelings about recent events, and responded to emotions expressed by others. Emotional expressions may have described current emotional states related to the novel disease or consequences of governmental measures, more free-floating daily or weekly moods (Oatley \& Johnson-Laird 2014, Russel 2003), as well as thoughts about recent and others' emotional experiences (see Sun, Schwartz et al., 2020, Galesic et al. 2021). Additionally, online emotional expressions may at least in part reflect people's cultural values (Hsu et al. 2021).

Literature on pandemics discusses anxiety, sadness, anger as well as positive emotions, although to a varying degree (e.g. Kim \& Niederdeppe, 2013, Jungmann \& Witthöft 2020, Meadows et al., 2019). Yet, it mostly adopts a clinical perspective (e.g. Jungmann \& Witthöft, 2020; Holman et al., 2020) or investigates individual emotions using mental health apps (Yarrington et al. 2021) or surveys (e.g. Holman et al., 2020; Pagnini et al., 2020; Sun, Balabanova et al., 2020). Preliminary social media analyses of emotions during COVID-19 exist only for specific cities in the US and for France (Ashokkumar \& Pennebaker, 2021; Castaldo et 
al., 2020; Valdez et al., 2020). They focused on tweets containing a few COVID-related keywords, and did not consider user locations or temporal changes during the outbreak (Castaldo et al., 2020; Lwin et al., 2020). To date, no study has documented the evolution of emotions from before the outbreak in more than a single country, and investigated their association with the spread of the virus and increasingly stringent governmental measures. In the following, we describe each emotion and its relevance to the COVID-19 outbreak.

Anxiety is a negative, arousing emotional state characterized by uneasiness and worry. It arises when people anticipate a threat, lack clear explanations, and feel unable to cope with the threat (Oatley \& Johnson-Laird, 2014, American Psychiatric Association, 2013). Anxiety influences compliance with recommendations (Bults et al., 2011; Rubin et al., 2009), risk taking (Lerner \& Keltner, 2001) and active information seeking (e.g. Kim \& Niedereppe, 2013). Anxiety should increase early on during a pandemic outbreak, as people start anticipating the threat from the virus and the consequences of governmental measures for their professional and social lives. We expected expressions of anxiety to increase with rising infection numbers, and in particular together with increases in the stringency of measures, which affected everyone's daily lives. Based on findings on the evolution of anxiety during H1N1, swine-flu and measles outbreaks (Bults et al., 2011; J. H. Jones \& Salathé, 2009; Meadows et al., 2019), we further expected anxiety to decrease after a couple of weeks.

Sadness occurs in response to losses, separation and unfulfilled goals (Oatley \& Johnson-Laird, 2014; Scherer, 1997). When people are sad, they turn inward, withdraw and slow down, and their thinking becomes more analytical, pessimistic, and biased towards negative aspects (Sander \& Scherer, 2009). The outbreak separated people from their friends and family and kept them from following their everyday life routines and hobbies, and sometimes entailed unexpected deaths. During a pandemic, people anticipate the threat of the novel disease and protection measures, before they are exposed to the consequences. We therefore expected sadness to increase more slowly than anxiety, as restrictions of everyday life and social contact were already in place.

Anger is a negative, arousing and action oriented emotion that motivates people to eliminate perceived obstacles (Frijda, 1986). It arises when people blame individuals or institutions for a negative event (Frijda 1986), can undermine trust in public institutions (e.g. Coombs, 2007, Kim \& Niedereppe, 2013), and reduce compliance with governmental regulations (Peitz et al., 2021). Predictions regarding anger in response to negative consequences from the outbreak were difficult, given that the virus itself is not an obvious target for anger. It was further unclear if people would blame or welcome governmental measures. Twitter is commonly used as a forum to discuss daily politics and other controversial issues. Exploring changes in anger expressions and their association with stringency of measures could thus reveal if people were more or less opposed to political decisions during the outbreak compared to before the pandemic.

Positive emotions do not entirely disappear during times of stress and might mitigate the negative impact of a crisis (Kim \& Niederdeppe, 2013). Nurturing positive emotions can contribute to foster trust and effective coping, reduce physiological arousal in the short-term and the risk of depression in the long-term (Fredrickson et al., 2003; Kim \& Niederdeppe, 2013). We tested if positive emotions would drop like common sense suggests, or persist as previously observed (Fredrickson et al., 2003; Kim \& Niederdeppe, 2013). For instance, Twitter users may 
have noticed positive aspects of the situation, expressed gratitude toward hospital staff, or expressed relief about their governments taking action.

Finally, we expected the emotional changes to be partly driven by collective dynamics between Twitter users. When writing a tweet or a reply that expresses an emotion, each user is likely to affect other users who in turn affect that user. In this way, conversations between people can continuously reactivate emotions, leading to collective emotions (Garcia \& Rimé, 2019; Goldenberg et al., 2020). During the unexpected COVID-19 outbreak, people were likely strongly motivated to share their thoughts and feelings with others. Many were further actively looking for information about how to cope with potential danger, and therefore possibly quite susceptible to other's influence. Abundant social sharing and strong emotional contagion should contribute to particularly strong and long-lasting emotional changes, especially when compared with the typical length of a purely individual emotional experience in isolation (e.g., Pellert, Schweighofer, et al., 2020).

\section{Methods}

\section{Materials}

To assess expressions of emotions in tweets, we used word lists for anxiety, anger, sadness and positive emotions from the Linguistic Inquiry and Word Count (LIWC) Dictionary. We used the 2015 version of LIWC in English (Pennebaker et al., 2015), and translations of the English 2007 version in French (Piolat et al., 2011), Dutch (Boot et al., 2017), German (Wolf et al., 2008), Spanish (Ramírez-Esparza et al., 2007) and Italian (Agosti \& Rellini, 2007). The aggregated text of all tweets for each language and country was summarized with word frequency statistics that were inspected by at least one fluent speaker of the language to check that no artifacts or word mismatches affected the analysis. We removed word list entries clearly related to the outbreak of COVID-19 (e.g. "deaths", "heal"), non-emotional country- and language-specific mismatches (e.g. city names), and common words mostly unrelated to the specific emotion (e.g. "opinion" ("avis") in the French sadness list, extremely ("äußerst") in the German positive emotions list). Additionally, we excluded common English positive words that negatively correlate with well-being and happiness at the regional level, namely "love", "good", "LOL", "better", "well" and "like" (Jaidka et al., 2020). Table S13 lists all exclusion terms. We test the robustness of the LIWC analysis by comparing with the results of a machine learning model for emotion categorization (Barbieri et al., 2020) based on a RoBERTa (Liu et al., 2019) supervised classifier trained with the annotated tweets from the SemEval dataset (Mohammad et al., 2018).

\section{Datasets}

Our analysis is based on a sample of 8,3 billion public tweets in six languages fluently spoken by at least one of the authors (English, Italian, Spanish, German, Dutch and French). Based on that list of languages, we selected countries from the list of countries with the most cases as of 1 April 2020, resulting in a list of 18 countries: Italy, France, Spain, the United Kingdom, Ireland, 
Germany, Austria, Switzerland, the Netherlands, Belgium, the United States, Canada, Australia, New Zealand, Peru, Ecuador, Chile, Mexico. For each country, we analyzed the language most spoken in the country, i.e. Dutch in Belgium, German in Switzerland, and English in Canada. We launched large-scale analyses to get aggregates over all tweets per day on the Forsight platform of the analytics company Crimson Hexagon (https://forsight.crimsonhexagon.com/), calculating exact counts of the daily number of tweets that contained at least one of the emotional terms of each list between 1 January 2019 and 15 April 2020 (see the Supplementary Information (SI) for more details on this measure). Our sample includes only original tweets (no retweets) from users located in the respective country by Crimson Hexagon. The location algorithm of Crimson Hexagon manages to match $90 \%$ of all posts in a country to a location using a combination of geo-coordinates, location information from user profiles, and users' time zones and languages ${ }^{1}$. We further excluded tweets by users with follower numbers below 100 and above 100,000 to remove spam and mass media (100 and 50,000 for countries with less than 15 million inhabitants: Austria, Belgium, New Zealand, Switzerland, see SI and Table S12 for a robustness check of the upper follower limit). The final sample of total tweets per country, including tweets with emotional expressions, contains between 4.7 million and 4.9 billion tweets per country (see Table S1). To contextualize our results in time, we obtained numbers of confirmed cases and deaths from the John Hopkins COVID-19 database (Dong et al., 2020). For stringency of measures, we used the stringency index from the Oxford COVID-19 Government Response Tracker (Hale et al., 2021).

To check the robustness and validity of our dictionary-based emotion measures and inspect the topics discussed in tweets, we analyzed two further types of data for a number of example countries: the 60 most frequent terms in tweets per emotion category, and the text of a random sample of tweets. For the first, we chose one example country per language (Germany, UK, Netherlands, France, Italy, Spain) and downloaded the relative frequencies of the 60 most frequent terms for tweets in general, and tweets in each emotion category. For the second, we downloaded a random set of at least 7,000 tweets per day for the US and Spain via the Crimson Hexagon API. Finally, to characterize general changes of activity on Twitter during the outbreak, we downloaded the total number of tweets and unique authors via the same platform for all countries (see SI for more details on these two data sets).

\section{Data analysis}

Data analysis was done in $\mathrm{R}$ version 3.6.1 (R Core Team, 2018), using the packages from the Tidyverse (Wickham et al., 2019), as well as Ime4, scales, cowplot, ggrepel and gridExtra (Auguie, 2017; Bates et al., 2015; Slowikowski, 2019; Wickham \& Seidel, 2019; Wilke, 2019). All $\mathrm{R}$ code and data are available at https://osf.io/736 kc/. Only the machine learning analysis for the robustness check was done in Python version 3.9.2, using packages Transformers (4.5.1) and Torch (1.8.1+cu102). Our analysis was not pre-registered.

Evolution of emotions over time. We calculated the daily percentage of tweets containing at least one word from the anxiety, anger, sadness or positive emotions word list for each country

1 https://web.archive.org/web/20210527091937/https://www.brandwatch.com/wpcontent/uploads/2020/10/CrimsonHexagon_Location_Methodology.pdf 
and emotion. Emotion levels were baseline corrected to make country-levels more comparable, and to correct for weekly fluctuations in the expression of emotions (i.e., lower levels during weekends). For this, we calculated the mean percentage per country, emotion and weekday. We then corrected all daily values in 2020 by subtracting and dividing by the corresponding baseline value. For example, anxiety levels in Spain on each Monday in 2020 were corrected with the mean percent of anxiety tweets in Spain on Mondays in 2019. The same procedure was used to assess percentage change of general activity on Twitter (followers, unique authors, total tweets).

We statistically assessed changes in emotion expression compared to the 2019 baseline across six periods: A period before the COVID-19 outbreak in any of the countries from 15 January to 15 February 2020 (control period), and five one-week periods covering the first weeks after the COVID-19 outbreak, starting on the day with 30 confirmed cases in each country. We first calculated the number of tweets in total and of tweets containing an emotion word across each of the periods. Second, we downsampled the data in the baseline and control period to the duration of the shortest time period (i.e., 7 days). Third, we used a generalized linear mixed logistic regression model fit by maximum likelihood approximation (Bates et al., 2015) for each emotion to predict if a tweet contained at least one emotion word. This approach allows capturing common changes in emotions across countries (fixed effects) on top of individual country variations (random effects). We entered time period with the baseline period as the reference level as a fixed-effect, and a random intercept and slope for time period per country to account for differences between countries (e.g. different baselines, languages, versions of the word lists), and for possible interactions between time periods and countries. To assess changes between time periods, we repeated the same analysis with contrasts comparing each period to the previous period instead of the baseline. 95\% confidence intervals for regression coefficients are Wald estimates.

Exploring possible drivers of emotions. To explore possible drivers of emotions which may explain part of the variation across countries, we calculated cross-country correlations of anxiety expression with confirmed cases per million inhabitants, and compared levels of all emotions in time periods with low, increasing and high measure stringency. We chose not to correlate anxiety expression with the number of deaths, as interpretation of this is difficult because deaths directly depend on confirmed cases, and are a lagged version of the number of cases. For caseanxiety correlations, we used Spearman-rank correlations to minimize the effect of single outliers in our relatively small sample of 18 countries. We first examined the association of average cases and anxiety expression across the five weeks after the outbreak, as well as weekly associations of the absolute case difference to the previous week with mean anxiety levels in the current week. We used absolute changes as cases were already corrected per capita and their number depends on the population. Furthermore, we considered it more plausible that people think in absolute rather than relative changes (e.g. "500 new cases this week" rather than "double the cases this week"). Time periods with different levels of measure stringency were defined based on the time series of the stringency index (see Supplementary Figure S6). We selected time periods with low, strongly increasing and constantly high stringency of measures, defining the first clear increase as a 20 point increase within 7 days (day 0). Specifically, these periods included a week-long period before the clear increase (day 14 to -7 ), one week around the clear increase (day -3 to +4 ), and a two-week period two weeks 
after the clear increase (day 14 to 28). We compared mean levels of emotion expression per country in each period to the previous period using a linear mixed-effects model with a random intercept per country. The period before the increase was compared to the control period (15 January to 14 February).

Duration of changes in emotional expression. As one potential indicator of the collective nature of the emotional responses we observed, we evaluated the duration of changes in emotions across the whole analyzed time period (1 January 2019 to 15 April 2020) by counting the number of days in a row during which an emotion remained consistently above or below a threshold of one median absolute deviation in 2019 away from the baseline. We chose this measure over the standard deviation to avoid biases in the threshold from outlier events in 2019. We then checked if the maximal number of days in a row for each emotion had occurred before or during COVID-19 based on the first confirmed case in each country.

Robustness check of emotion measures. For two example countries (USA, Spain), we checked the robustness of our dictionary-based emotion measures by comparing it with measures based on a machine learning model for emotion categorization. For this, we finetuned the pretrained masked language model from the TweetEval Benchmark (Barbieri et al., 2020) - a model based on RoBERTa-base (Liu et al., 2019) - on English and Spanish tweets from the SemEval dataset (Mohammad et al., 2018). We then correlated the percentage of emotional tweets based on dictionary-based (anxiety, anger, sadness, positive LIWC) and machine-learning based emotion labels (fear, anger, sadness and joy + optimism + love predicted by RoBERTa). We counted a tweet as emotional if the model predicted a probability higher than 0.9 for the specific category, which is a conservative threshold to ensure high precision.

Word frequencies. Furthermore, we contextualized LIWC words using word frequency plots for the 60 most frequent terms in tweets per emotion category for six example countries (one per language). We compared word frequencies during the baseline and the five weeks after the outbreak, inspecting which topics people were emotional about (i.e. frequent non-LIWC terms), and the most frequent LIWC terms. Here, disproportionally large contributions by single words would indicate a problem, for instance in the case of a single event strongly impacting our tweet count without necessarily reflecting a general change in emotions. In particular, some otherwise unexplained outliers among non-LIWC words might indicate frequent usage of LIWC terms in an irrelevant context. The word frequency plots S2.1-S2.12 and Tables S2.1-S2.4 in Su 2 (SI 2) show that there were no such cases.

\section{Results}

An interactive visualization of all results can be found at http://dgarcia.eu/COVID19Emotions.html. Table S1 presents the grand mean of percentages of tweets containing emotion words per country. During baseline, $9.80 \pm 2.97 \%$ (mean \pm SD) of tweets contained anxiety expressions, $3.57 \pm 1.04 \%$ contained sadness expressions, and $5.98 \pm 0.74 \%$ contained anger expressions, and $44.77 \pm 16.14 \%$ contained positive expressions, respectively (see Table S2). All results focus on changes from the respective baseline per emotion and country. 


\section{Evolution of emotions over time}

Figure 1 depicts the evolution of the percentage of tweets containing terms related to anxiety, anger, sadness and positive emotions in 2020 in comparison to the baseline in 2019 in four exemplary countries, selected based on the highest number of confirmed cases in our sample on April 15, 2020 (the end of our analysis period). We labeled peaks as specific events based on the most frequent terms in tweets from the given day(s) using word clouds. We additionally read example tweets containing these terms, and verified the event they referred to by checking

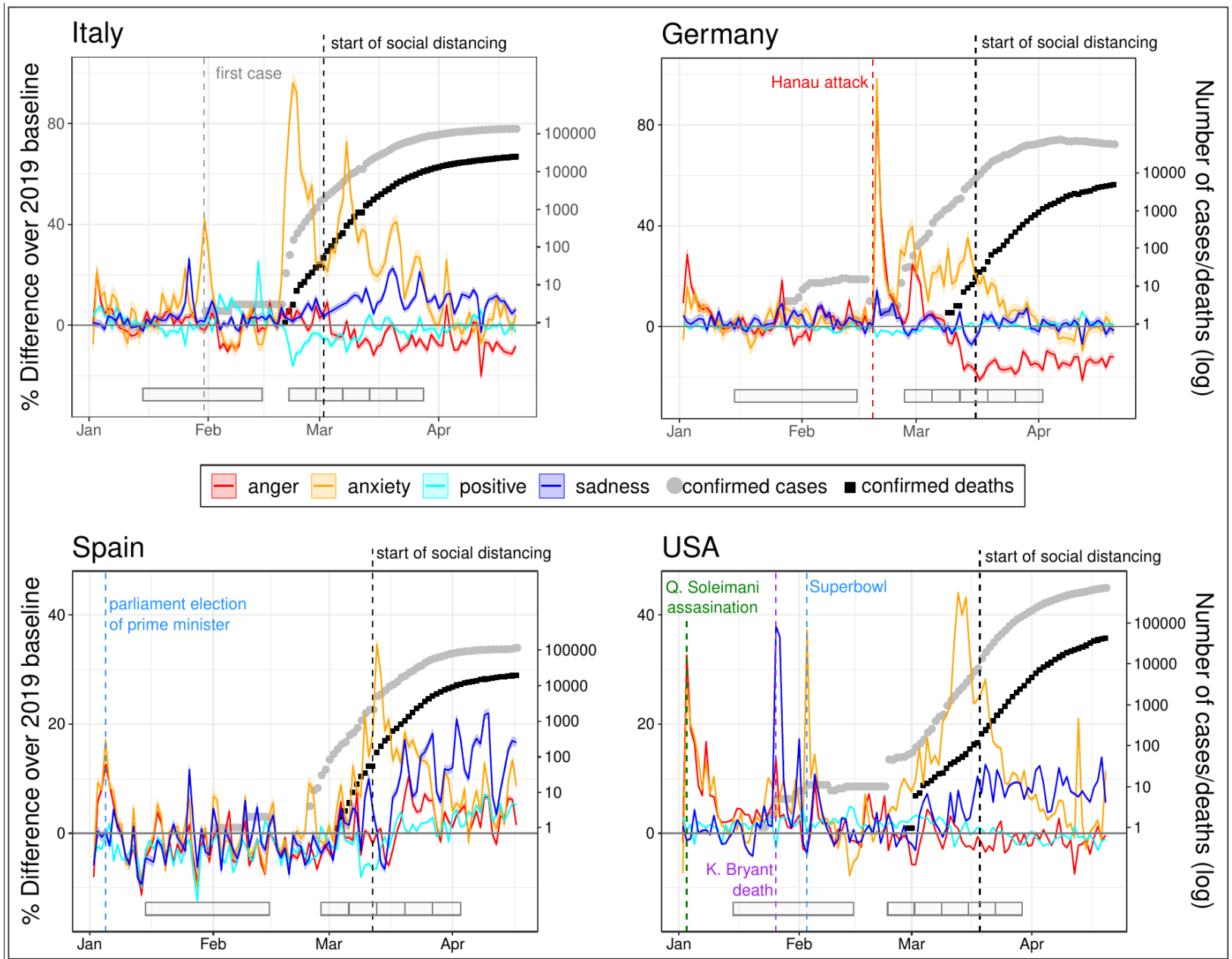

Figure 1. Time series of the level of anxiety, anger, sadness and positive emotion expression compared to the baseline in four selected countries. The left $y$-axis depicts the percentage of tweets containing words for each emotion. A value of zero corresponds to the average level per weekday in 2019. The secondary $y$-axis depicts cumulative number of cases and deaths on a log-scale (the maximal number is different for each country). Note that the primary y-scale displays different maximal values for different countries to clearly show within-country changes for each emotion (see discussion for a cautionary remark on between-country comparisons). Colored vertical lines depict important events for the respective day, which were identified using word clouds for tweets on the respective day. The vertical black dashed line indicates the onset of nationwide social distancing measures concerning a majority of the population. The gray rectangles at the bottom indicate the time periods used for the regression analysis depicted in Figure 3 (control period, and 5 one-week periods after the day with 30 confirmed cases in the respective country). Shaded areas are binomial $95 \%$ confidence intervals calculated with the 
Clopper-Pearson method (very narrow for these four countries due to large sample sizes of tweets).

online news reports. To enrich interpretation of timelines, starting dates of nationwide social distancing measures concerning the majority of the population retrieved from Desvars-Larrive et al.(2020) were added to these figures. Such measures included closures of kindergartens, schools, bars, restaurants and shops, prohibition of small gatherings, lockdowns and quarantines. For the USA, we chose the date on which the first state implemented such measures. Supplementary Figures S1 and S2 depict emotion timelines for all other countries, and Table S2 provides averages per time period. Figure S3 shows separate emotion time series for two US states with early severe outbreaks (California and New York), showing similar trajectories as the rest of the US.

Anxiety. After the outbreak of COVID-19, defined as the day on which confirmed cases reached 30 in each country, the percentage of tweets containing anxiety-related words increased in all countries (Figure 2a illustrates the size of increase per country, averaged across the five weeks after the outbreak). In most countries, including the four shown in Figure 1, anxiety terms on Twitter strongly increased in the early phase of the outbreak. The results of a mixed-effects logistic regression model (see Figure 3a) reveal that anxiety expression was already slightly elevated compared to baseline during the control period from mid-January to mid-February $(\beta=$ $0.02,95 \% \mathrm{Cl}=[0.01,0.04], \mathrm{z}=2.85, \mathrm{p}=.004$, OR $=1.02$ ), a time during which first cases occurred in 8 out of 18 countries. However, levels abruptly and strongly increased in week 1 (control - week 1: $\beta=0.18,95 \% \mathrm{Cl}=[0.12-0.24], z=5.63, p<.001$, OR = 1.19) to a much higher level $(\beta=0.2,95 \% \mathrm{Cl}=[0.13,0.27], z=5.84, p<.001, \mathrm{OR}=1.22)$, at which they remained until week $3(\beta=0.22,95 \% \mathrm{Cl}=[0.16-0.28], z=6.97, p<.001, \mathrm{OR}=1.24)$. In week 4 and 5 , the proportion of anxiety terms strongly decreased again compared to the previous week (week $3-4: \beta=-0.09,95 \% \mathrm{Cl}=[-0.11,-0.06], z=-7.16, p<.001, \mathrm{OR}=0.92$; week $4-5: \beta=$ $-0.05,95 \% \mathrm{Cl}=[-0.07,-0.03], z=-4.22, p<.001$, OR $=0.95$, see Table S4 for other contrasts against the previous week). Nevertheless, anxiety expression remained significantly above the baseline throughout all 5 weeks after the outbreak (see Table S3 for all contrasts against the baseline). Inspecting the anxiety time series throughout 2019 and 2020 revealed that anxiety expressions reached their highest level during the outbreak in all but Latin American and German-speaking countries. In the latter, a higher but short anxiety peak in response to a terrorist attack in Hanau on 25 February 2020 reflects the high frequency of the word "terror", which is part of the anxiety and anger word lists.

Sadness. All but two countries also experienced significant increases in tweets containing sadness-related terms after the outbreak, and none experienced a decrease (Figure $2 b$ ). However, in comparison to anxiety, sadness terms rose more gradually and remained close to their highest level until the end of the analyzed time period (see Figure 3b, Table S5 for contrasts with baseline, Table S6 for contrasts with the previous period). At a level just above the baseline in the control period $(\beta=0.02,95 \% \mathrm{Cl}=[0.00,0.03], z=2.23, p=.026, \mathrm{OR}=1.02)$, they did not increase significantly in week 1 (control - week 1 : $\beta=0.02,95 \% \mathrm{Cl}=[-0.01,0.04]$, $z$ $=1, p=.315, \mathrm{OR}=1.02$ ). From there, they increased markedly in two steps (week $1-2: \beta=$ $0.05,95 \% \mathrm{Cl}=[0.02,0.08], z=3.59, p<.001, \mathrm{OR}=1,05$; week $2-3: \beta=0.03,95 \% \mathrm{Cl}=[0$, $0.06], z=2.25, p=.022, O R=1.03$ ) until reaching their highest level in week 3 and 4 (week $3: \beta$ $=0.12,95 \% \mathrm{Cl}=[0.07,0.16], z=5.10, p<.001, \mathrm{OR}=1.12$, week $4: \beta=0.12,95 \% \mathrm{Cl}=$ 
$[0.09,0.15], z=8.51, p<.001, O R=1.13$ ). In week 5 , they decreased marginally again (week 4 $5: \beta=-0.01,95 \% \mathrm{Cl}=[-0.03,0.00], z=-1.99, p=.048, \mathrm{OR}=0.99)$. Although sadness terms occurred in less tweets than anxiety terms, their frequency was significantly higher than the baseline in all 5 weeks after the outbreak across countries (see Table S5 and confidence intervals in Figure 1, S1 and S2), and remained higher in 16 countries in week 5.

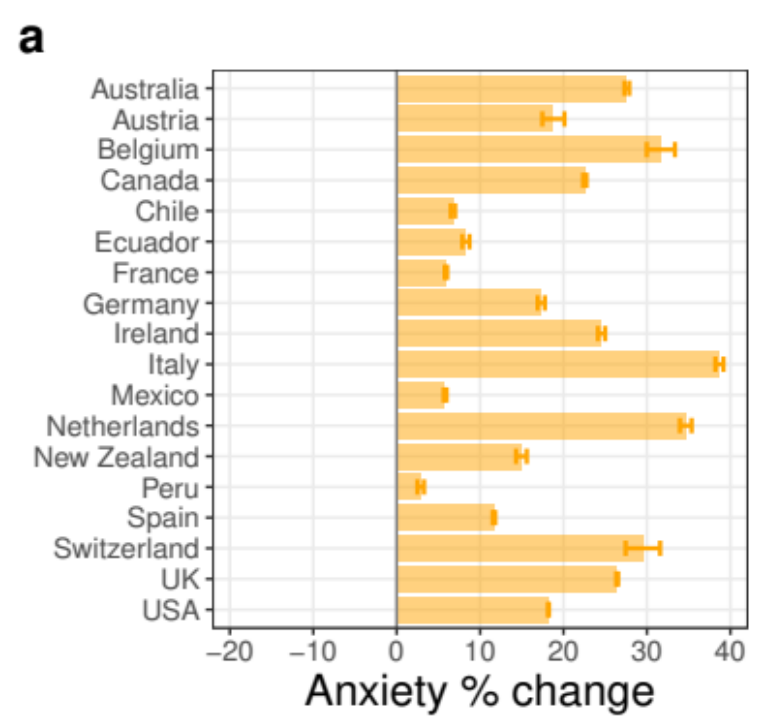

b

C
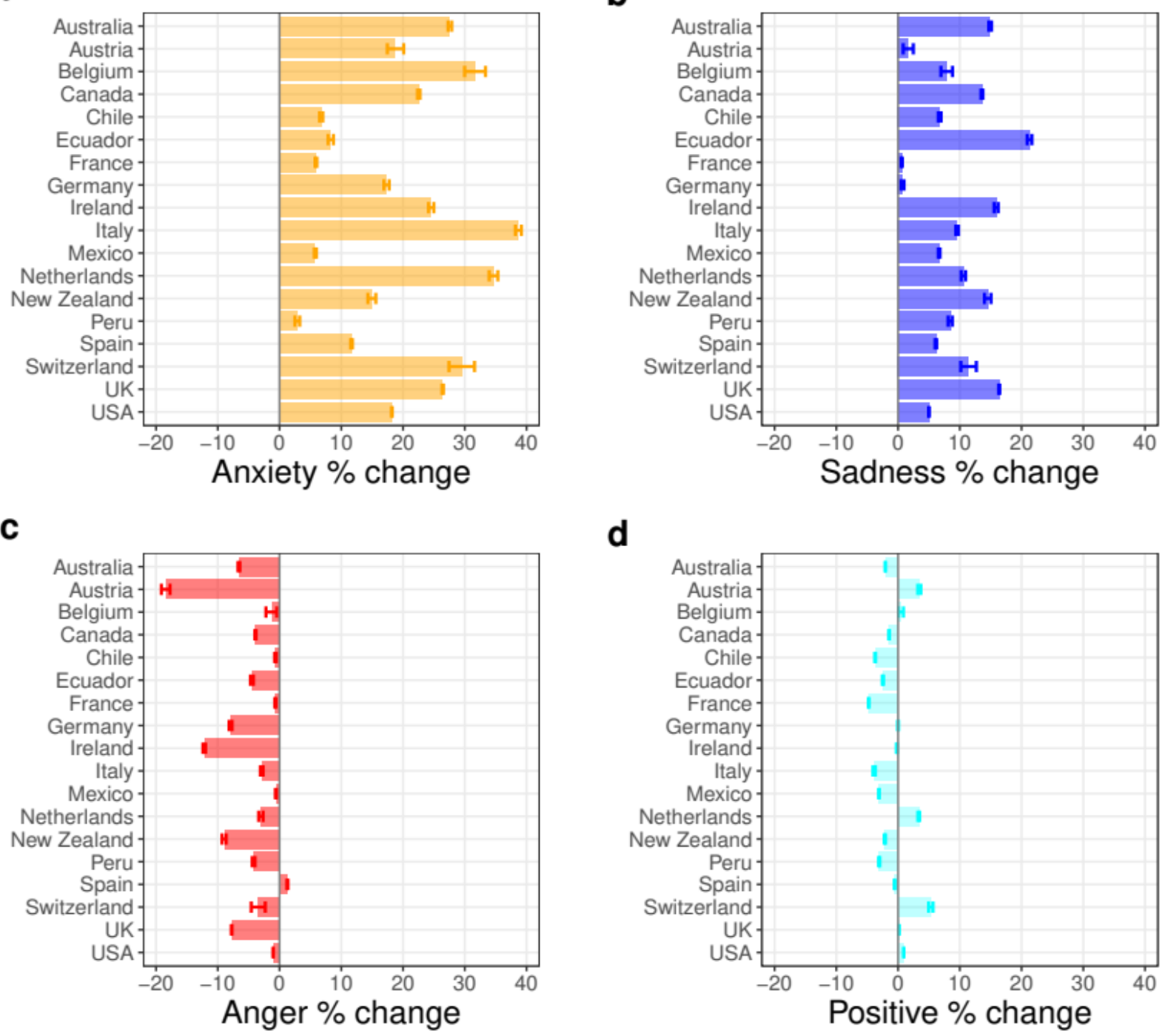

d

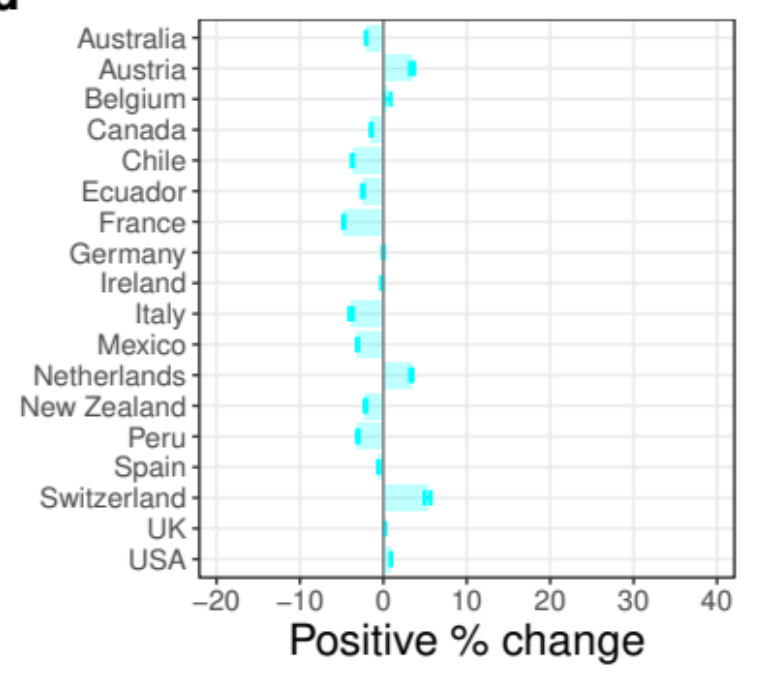

Figure 2. Consistency of emotion changes across countries in the 5 weeks after the outbreak. The $x$-axis depicts the average percentage change of tweets containing at least one emotion word in the five weeks after the COVID-19 outbreak compared to the baseline. Countries are ordered by language and continent. The start of the outbreak is defined as the date with 30 confirmed cases in each country. Error bars represent binomial 95\% confidence intervals calculated with the Clopper-Pearson method. a) All countries show a clear significant increase in tweets with anxiety terms. b) All but two countries show a significant increase in sadness expression, none shows a decrease. c) All but 4 countries show a significant decrease in anger expression, only one an increase. d) Changes in tweets with positive emotion words are small and inconsistent (increase in 3, decrease in 9, no significant change in 6 countries). 

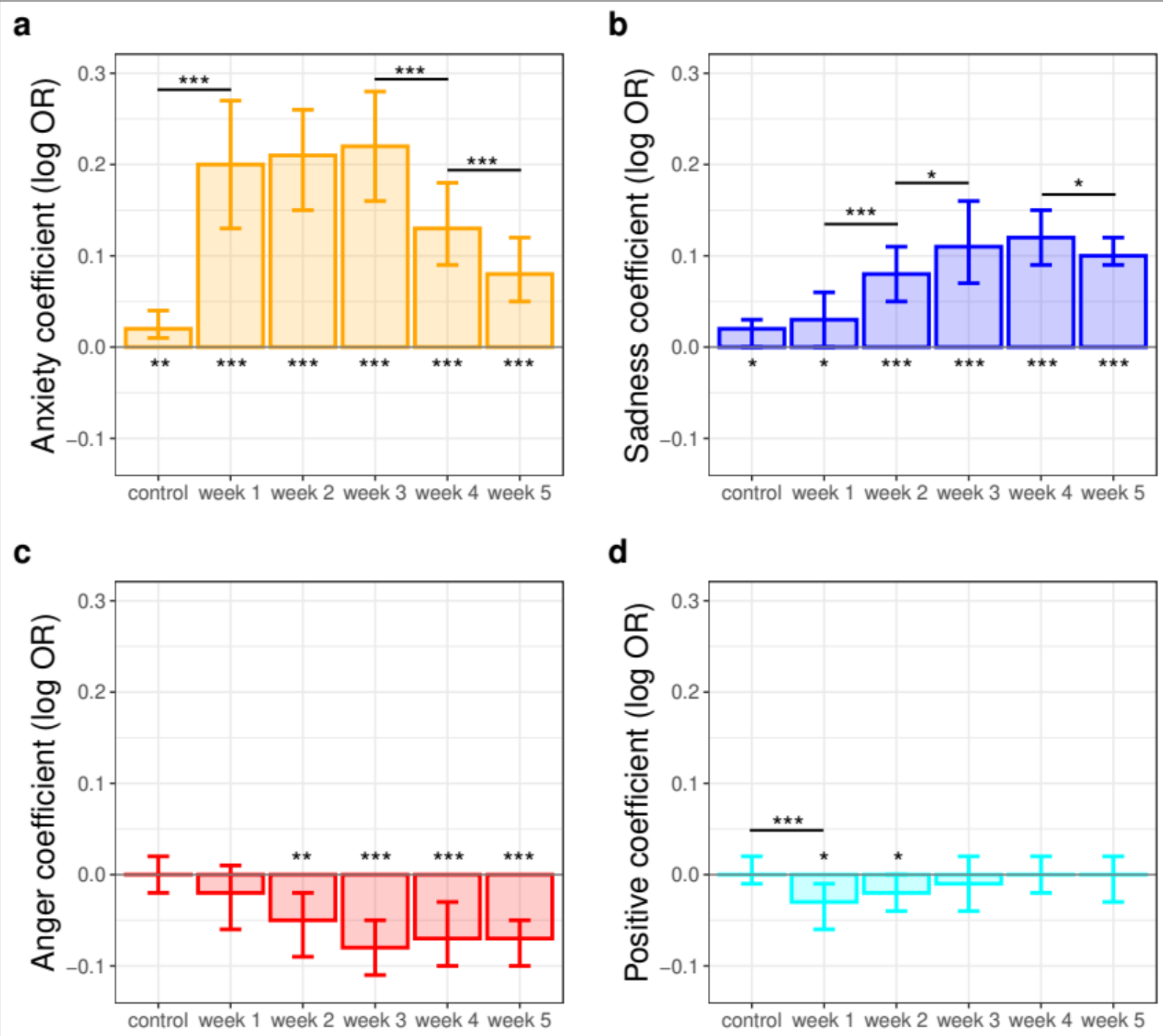

d

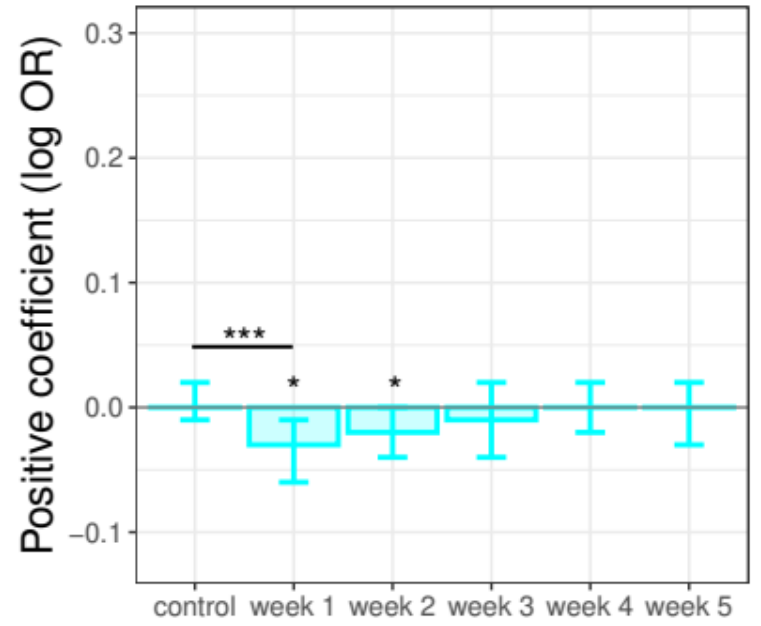

Figure 3. Changes of the probability that a tweet includes at least one emotion word across the six analyzed time periods. Bars and error bars represent fixed-effect regression coefficients with Wald confidence intervals across 18 countries.

Anger. In contrast to anxiety, and similar to sadness, changes in anger expressions were gradual rather than sudden, with strongest changes occurring in week 3 - 5. Twitter users started expressing less anger in 12 out of 18 countries around the onset of the broad social distancing measures (see Figure S1-S2). In eight of these countries, anger-related terms already diminished earlier, at a time where governments started taking actions against the virus and some first specific and limited social distancing measures were implemented. In total, anger terms decreased or remained stable in 17 out of 18 countries (see Figure 2c). In most countries experiencing a decrease, it remained below the baseline until the end of the analyzed time period. Logistic regression results (see Figure 3c) mirrored this result pattern (see Table S7 for contrasts against baseline, Table S8 for contrasts against previous period). In the control period, the proportion of anger-related tweets was not different from the baseline (control: $\beta=0,95 \% \mathrm{Cl}$ 
$=[-0.02,0.03], z=0.16, p=.869, O R=1)$. It then decreased in three small incremental steps (Table S8), reaching a level significantly lower than the baseline only from week 2 onward (week 1: $\beta=-0.02,95 \% \mathrm{Cl}=[-0.06,0.01], z=-1.31, p=.190, \mathrm{OR}=0.98$; week 2: $\beta=-0.06$, $95 \% \mathrm{Cl}=[-0.09,-0.02], z=-3.07, p=.002, \mathrm{OR}=0.95)$. It arrived at its lowest level in week $3(\beta$ $=-0.08,95 \% \mathrm{Cl}=[-0.11,-0.05], z=-5.07, p<.001, \mathrm{OR}=0.92)$, where it remained stable until the end of the analyzed period (Table S7).

Positive emotions. In contrast to negative emotions, the frequency of tweets with positive words remained relatively stable throughout the COVID-19 outbreak, and changes were less consistent across countries. Specifically, such tweets decreased and increased slightly in nine and three countries, respectively (see Figure $2 \mathrm{~d}$ ). Logistic regression coefficients across countries (Figure 3d, Table S9 for contrasts against baseline, and Table S10 for contrasts against previous period) revealed a small decrease early on (control - week 1 : $\beta=-0.04,95 \% \mathrm{Cl}$ $=[-0.06,-0.01], z=-2.71, p=.007, O R=0.96)$, lasting for the first two weeks after the outbreak (week 1: $\beta=-0.03,95 \% \mathrm{Cl}=[-0.06,-0.01], z=-2.35, p=.019, \mathrm{OR}=0.97$, week $2: \beta=-0.02$, $95 \% \mathrm{Cl}=[-0.04,0.00], \mathrm{z}=-2.16, \mathrm{p}=.031$, OR $=0.98)$. Levels in week 3 to 5 were not significantly different from the 2019 baseline (Table S9).

In summary, levels of tweets containing emotional expressions were similar to the 2019 baseline for all emotions during the control period from 15 January to 14 February 2020. After the COVID-19 outbreak, we observed early and strong increases in anxiety terms, followed by a later and more gradual increase of sadness- and decrease of anger-related terms. While anxiety-related terms decreased from three weeks after the outbreak onward, levels of sadness and anger-related terms remained relatively stable until the end of the observation period in week five. In many countries, changes in sadness and anger expression as well as the decrease in anxiety expression coincided with the onset of broad social distancing measures.

In addition to changes in the frequency of emotional expressions, overall Twitter activity increased as well in all countries. Not just the total number of tweets increased by about $50 \%$ with peaks of up to $120 \%$ in some countries, but also the total number of people posting tweets increased in all countries (see Figure S4).

\section{Exploring possible drivers of emotions}

Correlation of emotions with confirmed cases. In the 5-week period after the outbreak, there was a clear positive correlation of anxiety terms with cases per million ( $r s[18]=0.46, p=.058$, see Figure $4 a$ ). The strength of the correlation varied across the five weeks (see Figure S5), being strongest in week $1(\mathrm{rs}[18]=0.54, p=.023$, see Figure $4 \mathrm{~b})$, and then decreasing in the following weeks (week 2: $r s[18]=0.17, p=.503$, week 3: $r s[18]=0.047, p=.863$, week 4: rs[18] $=0.197, p=.446)$, except for week $5(r s[18]=0.45, p=.063)$. 

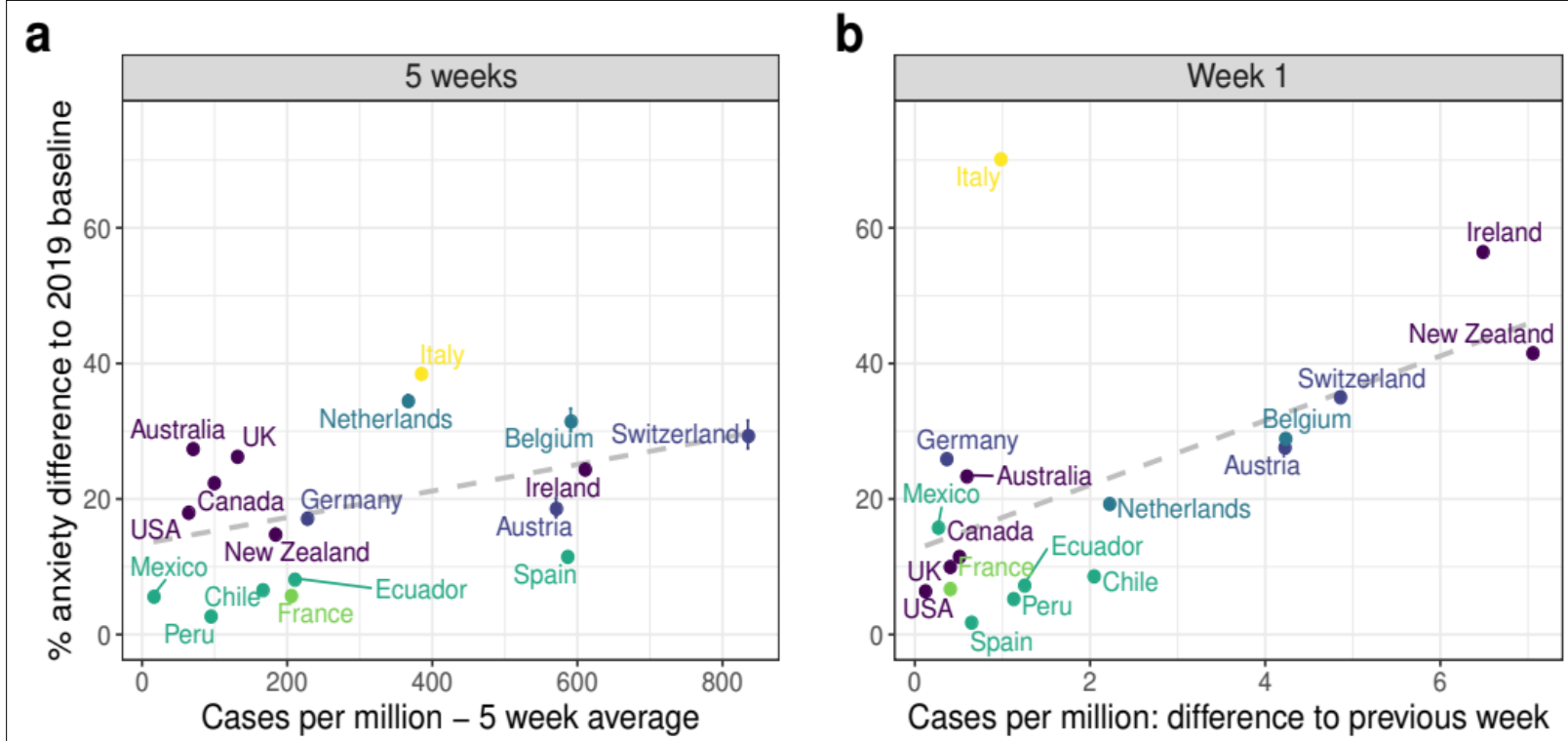

Figure 4. Cross-country correlation of mean changes in anxiety compared to baseline with mean confirmed COVID-19 cases: a) average of cases in the 5 weeks after the outbreak, (b) absolute difference in cases to the previous week, for the first week after the outbreak. Countries are coloured by language.

Measure stringency. Most countries had already implemented their most stringent measures by week 3 after the outbreak, keeping them throughout week 4 and 5 (see Figure $5 a$ for one example country, Figure S6 for all countries). The timeline of emotions and stringency measures reveal strikingly parallel developments: anxiety terms increase shortly before or at the same time as more stringent measures are implemented in 15 of 18 countries, sadness terms increase at the same time or shortly after in 15 countries, anger terms drop exactly as sadness terms increases in 12 countries, and positive expressions drop slightly and briefly at the moment that measures become more strict in 5 countries (Peru, Italy, New Zealand, Mexico, Chile). Anxiety terms start to decrease once stricter measures are in place. Statistical comparisons of time periods with low, clearly increasing and constantly high measure stringency mirrored these patterns (see Figure 5b): One week before the strong increase, anxiety terms were already significantly higher than during the control period $(b=0.12,95 \% \mathrm{Cl}[0.05,0.19], t(54)=3.32, p=$ $.002)$. Yet, it increased most strongly during the week of the first strong increase in stringency $(b=0.19,95 \% \mathrm{Cl}[0.11,0.26], t(54)=5.09, p<.001)$, and decreased again to a much lower level when stringent measures were in place for a while $(b=-0.24,95 \% \mathrm{Cl}[-0.31,-0.16], t(54)=-$ $6.51, p<.001)$.

All other emotions had not changed much one week before the clear increase in strigency (control vs. 1 week before: sadness: $b=0.00,95 \% \mathrm{Cl}[-0.05,0.05], t(54)=0.01, p=.990$ : anger: $b=0.01,95 \% \mathrm{Cl}[-0.03,0.04], t(54)=0.41, p=.685 ;$ positive: $b=0.00,95 \% \mathrm{Cl}$ $[-0.02,0.01], t(54)=-0.32, p=.753)$. They only started to change significantly when measures became stricter ( 1 week before vs. increasing stringency: sadness: $b=0.05,95 \% \mathrm{Cl}[0.01,0.1]$, $\mathrm{t}(54)=2.3, \mathrm{p}=.026$, anger: $\mathrm{b}=-0.08,95 \% \mathrm{Cl}[-0.11,-0.05], \mathrm{t}(54)=-4.61, \mathrm{p}<001 .$, positive: $\mathrm{b}=$ $-0.02,95 \% \mathrm{Cl}[-0.04,-0.01], \mathrm{t}(54)=-3.06, \mathrm{p}=.003)$. Two weeks later, sadness expression remained high (increasing stringency vs. 2 weeks later: $b=0.04,95 \% \mathrm{Cl}[-0.01,0.08], t(54)=$ $1.75, p=.086)$ and anger expression remained low $(b=0.02,95 \% \mathrm{Cl}[-0.02,0.05], t(54)=0.94$, 
$\mathrm{p}=.352)$, whereas positive expressions had recovered $(\mathrm{b}=0.03,95 \% \mathrm{Cl}[0.01,0.04], \mathrm{t}(54)=$ $3.25, p=.002)$.

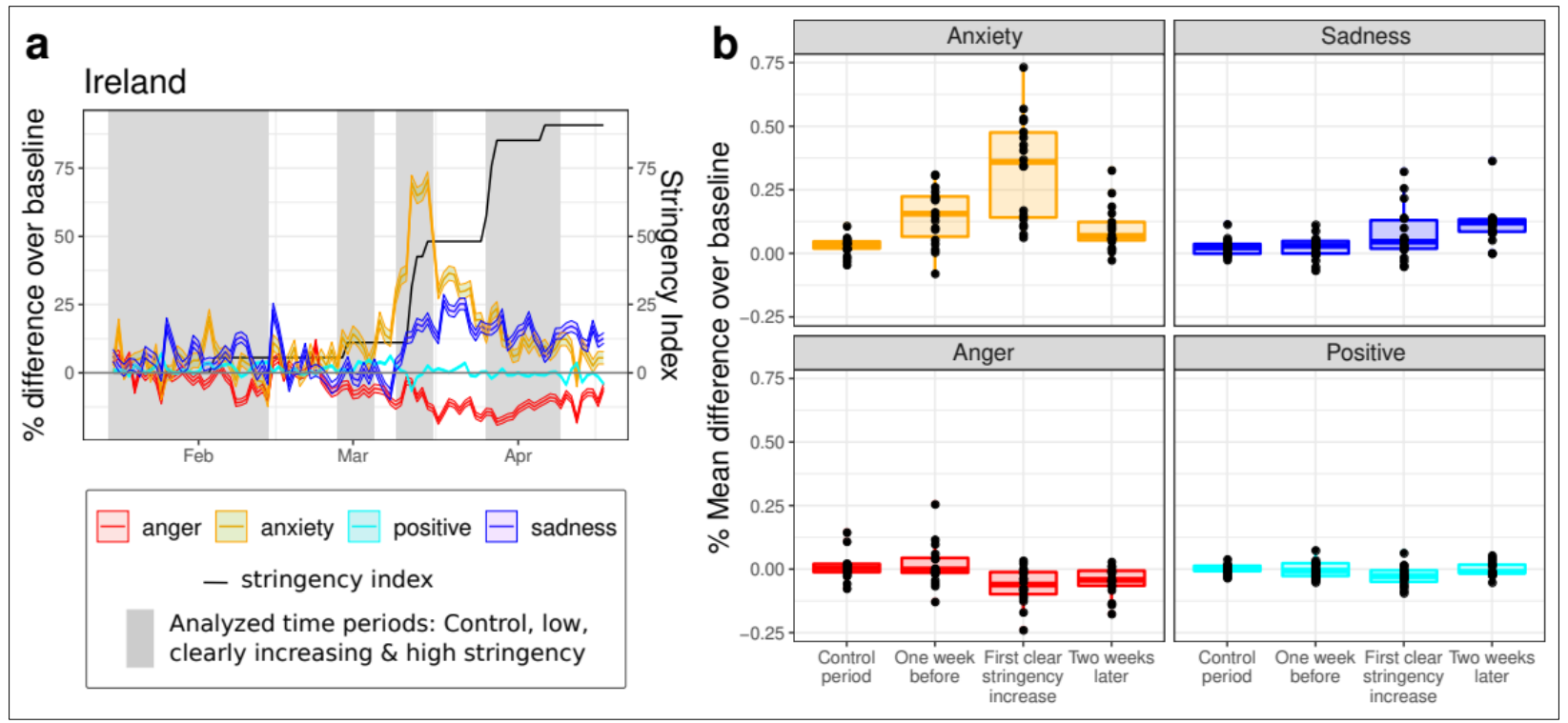

Figure 5. Emotion levels in time periods around the increase of measure stringency (a) Emotions and stringency time series in Ireland as one example country, indicating the control period, the period one week before, around and two weeks after the first clear stringency increase in gray. In the control period, there were none or only light measures in most countries. One week before the first clear increase, measure stringency was still low in most countries, two weeks after the first clear increase it was constantly high in all countries. (b) Boxplots of country emotion means in these time periods. Dots represent individual countries.

\section{Duration of changes in emotional expression}

When collective emotions arise, social interaction between users prolongs the time for which emotion expression occurs at a different rate, as individuals emotionally respond to the expressions of others. The maximal duration of sustained changes in all four emotions during COVID-19 were among the longest ones since the beginning of 2019 in all countries, and the single longest one in the majority of countries. Specifically, 16 out of 18 countries had not experienced such long periods of elevated anxiety and sadness expression before COVID-19 (see Figure 6). Ten and eleven countries, respectively, experienced the longest sustained periods of changed anger and positive expression during COVID-19 (see Figure S7). Longer increased anxiety expression before COVID-19 occurred only in two countries during political protests in 2019 (against social inequality in Chile, and austerity measures in Ecuador). Similarly, longer elevated sadness expression occurred in Chile during the same protests, and after a political scandal in Austria (the so-called Ibiza affair). 


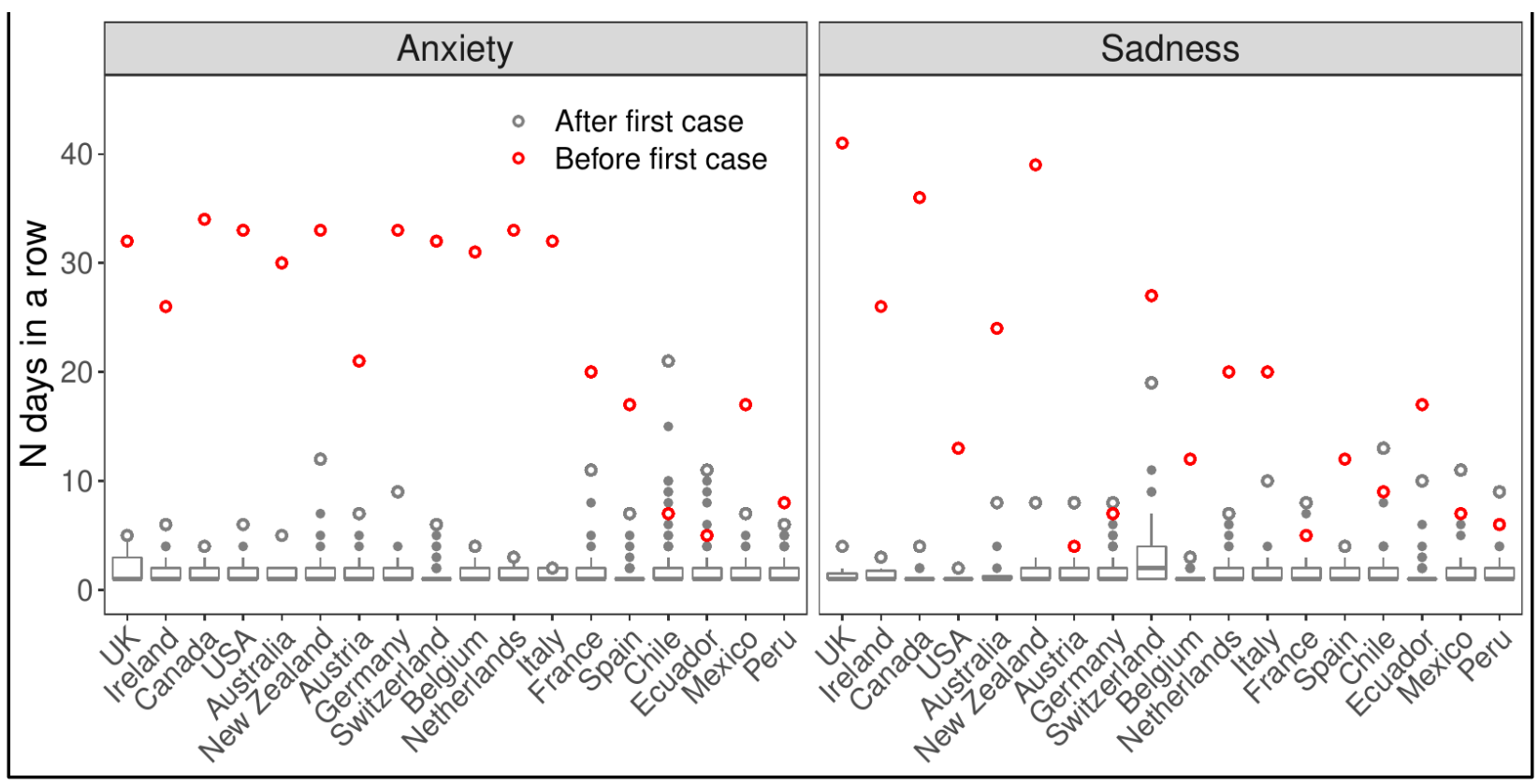

Figure 6. Time intervals for which tweets containing anxiety and sadness expressions remained continuously above their median frequency in 2019 in each country. Time intervals (number of days) are split into those occurring before (from 1 January 2019 until first case) vs. during the COVID-19 pandemic (from the first case per country until April 15). Empty circles represent the maximal number of days per time period, while the gray box plot represents the distribution of time intervals before COVID19. Because most countries only experience one or a few long intervals after the first case, only maximal values are depicted for this time period. Countries are ordered by language and continent.

\section{Robustness checks}

Correlations of machine-learning based (RoBERTa) and dictionary-based (LIWC) percentages of emotional tweets per day were strong and positive for all emotions. They were particularly high for anger, which could be expected given that both LIWC and machine-learning labels are meant to assess anger (US: $r=.73, t(475)=23.48, p<.001$, Spain: $r=.70, t(472)=21.07, p<$ .001 . For anxiety, they were slightly lower (US: $r=.64 \mathrm{t}(475)=18.1, p<.001$, Spain: $r=.37$, $t(472)=8.75, p<.001)$, possibly because the model predicts fear rather than anxiety labels. $A$ similar explanation applies to the correlation of LIWC positive emotion volumes and combined joy, optimism and love labels from RoBERTa (US: $=.42, \mathrm{t}(475)=10.21, \mathrm{p}<.001$, Spain: $r=.64$, $\mathrm{t}(472)=18.22, \mathrm{p}<.001)$. The correlation for sadness was high in the US $(r=.71, t(475)=$ 21.84, $p<.001)$, and moderate in Spanish $(r=.42, t(472)=10.06, p<.001)$. These high correlations indicate good convergent validity of LIWC with a state-of-the-art machine learning model (see Table S11 for the ROC curve for RoBERTa per emotion and language, where AUCs for English are around 0.9 and for Spanish around 0.8).

In addition to this robustness check of our measures using an alternative method, visual inspection of the spikes in response to other events speaks for the the face validity of our measures: For instance, in the US and Canada, tweets with sadness terms increased after reports about Kobe Bryant's death, whereas anxiety terms increased as Americans excitedly followed the play of their favorite football teams during Superbowl, and anger and anxiety 
expression spiked after the US military assassinated Iranian military officer Soleimani (see Figures 1 \& S2).

\section{Word frequencies}

Inspection of the most frequent terms in all tweets, and in tweets containing emotional words, revealed no particular words that were frequently used in a context irrelevant to the subject of this study. Furthermore, each frequent LIWC word only occurred in a small percentage of tweets, with a median of $1.17 \%$, and maxima during baseline and outbreak for shame (8\%) and good (3.8\%; see Tables S2.1-S2.4 for frequencies of other LIWC terms among the 60 most common terms). This suggests that the percentage of emotional tweets per category likely reflects the sum of a broadly distributed higher occurrence of emotional expressions, instead of single words having a strong effect on the change in each emotion measure. For instance, LIWC anxiety terms in French tweets occur in a maximum of $1.43 \%$ of tweets, the most frequent being inquiète (worried, 1.43\%), lâcher (letting go, 1.29\%), dérange (disturb, 1.15\%), lâche (coward, 1.12\%), énerve (nervous/irritated, 1.05\%), and hésite (hesitate, 1.01\%). Examples of other LIWC terms that made it into the top 60 in some of the countries, but all occurred in less than $2.4 \%$ of tweets expressing each emotion, are: (1) panic, fear, anxiety, pressure and stress in anxiety tweets; (2) isolating, disappointed, failure, tired, (I am) sorry, hurt, miss and useless in sadness tweets; (3) different swear words and mean, ridiculous, fight in anger tweets; and (4) smileys, happy, funny, heart, easy, beautiful, luck, and thank you in positive tweets.

Regarding the topics of conversations in which emotional expressions occurred, word frequencies reveal several trends that are visible across countries and emotions: First, the nonemotional words reveal a shift of topic discussed in emotional tweets from politics during baseline (typical terms: party, politician and country names, Brexit, government) to topics associated with the pandemic during the outbreak (coronavirus, virus, covid, measures, crisis etc.) and the related everyday life changes (home, people, social distancing terms). During the outbreak, all kinds of tweets more often mentioned people (and similar terms like children, family, staff, women), home, work, life, hope, and time (and similar terms like day, today, week). Second, words related to social distancing (quarantine, isolation, confinement, social, lockdown, stay at home, going out) often rank higher not only in sadness tweets, but also other emotional tweets, compared to tweets in general. Positive emotion tweets also frequently contained words like home, books, team, family, hug etc. Detailed results for six example countries are reported in SI 2.

\section{Discussion}

This study investigated collective emotions during the early COVID-19 outbreak in 18 countries by analyzing digital traces of changes in the frequency of emotional expressions in a massive Twitter data set. We observed a strong upsurge of anxiety-related terms in tweets at the beginning of the pandemic in all considered countries, which was related to the growth in cases and increases in the stringency of governmental measures. About two weeks later, sadness- 
related tweets increased in parallel with simultaneous decreases in anger-related ones in most countries. In contrast to negative emotions, we found only a small decrease in words related to positive emotions when measures became more stringent, and this decrease did not occur in all countries. Strikingly similar patterns for anxiety, sadness and anger expressions have been reported recently in an analysis of Reddit comments from eight US cities during the COVIDoutbreak (Ashokkumar \& Pennebaker, 2020). Furthermore, the relative stability of positive expressions is consistent with observations from previous crises (Fredrickson et al., 2003; Kim \& Niederdeppe, 2013).

The initial rapid increase in tweets with anxiety terms, and the long period during which they remained above the baseline level may indicate that social sharing of emotions (Rimé, 2009) amplified the response to the impending threat. Words like staying (at home), buying (of groceries), emergency, health, contagion and information were among the most frequent words in anxiety tweets (see SI2), suggesting that a large proportion of people's worries was directly linked to the pandemic. Anxiety terms mostly started to increase shortly before or together with an increase in the stringency of measures. Only in Austria and Switzerland, anxiety terms increased around a month earlier, hinting that people in these countries anticipated pandemic consequences earlier, possibly due to news coverage about the severe outbreak in the nearby Italian region Lombardia. The increase in anxiety terms was accompanied by one in activity on Twitter in general, consistent with its function to motivate active information seeking (e.g. Kim \& Niedereppe, 2013). During the start of the outbreak, anxiety expression was associated with the growth in cases across countries, driven by around six countries with strong increases. In countries with local hot-spots or overall highest case numbers in the analyzed period, the term cases was among the most frequent in anxiety and sadness tweets (France, Italy \& Spain, see SI2).

In all countries, anxiety expressions reduced after the most stringent measures (lockdowns) had been implemented. This decrease about three weeks after the outbreak is consistent with findings during the H1N1, swine-flu and measles outbreaks (Bults et al., 2011; J. H. Jones \& Salathé, 2009; Meadows et al., 2019). It could indicate that people, initially worried about the disease and the measures, were reassured once measures were in place and provided a sense of coping and protection. In addition, conversations about a crisis event also fade as people become satiated with other people's emotional expressions (Pennebaker \& Harber, 1993). This decline was more gradual than after one-off disasters such as terrorist attacks or earthquakes (Garcia \& Rimé, 2019; Gruebner et al., 2017), possibly due to the more diffuse and temporally distributed nature of the threat.

Tweets with sadness terms rose more gradually and after anxiety terms and then remained relatively stable until five weeks after the outbreak. This makes sense as exposure to threat precedes exposure to losses (social restrictions, casualties, economic consequences) during a pandemic. Sadness expressions increased in almost all countries, and most commonly after more stringent measures were implemented, remaining relatively stable afterwards. This highlights social distancing measures as a major driver of this emotion. Consistent with this interpretation, words related to social distancing (quarantine, isolation, confinement, social, lockdown, stay at home, going out) were used more often in tweets expressing sadness (and other emotions) than in tweets in general. In contrast, deaths were only featured among the 
most frequent terms in France (out of the six countries for which we performed the respective analysis). Altogether, their time course and the topics in sadness tweets hint that changes to people's everyday life, rather than deaths, were a driving factor.

Decreases of anger expressions around the onset of measures may indicate both that people were overall not opposed to them, and that the usual discussions on other controversial political topics were scarce in the early phase of the COVID-19 outbreak. A general shift in topics of conversation from political ones in 2019 to pandemic-related issues after the outbreak (see SI2) is visible across countries and emotions, and supports this interpretation. Although media discourse might have created the impression that people were angry about government regulations during the first outbreak, they may actually have been less angry than about previous political decisions. The low level of anger expressions remained less frequent throughout the period of high measure stringency, highlighting the potential role of social distancing measures for anger expressions. Positive expressions mostly remained at their relatively high baseline level, only decreasing slightly in about half of the investigated countries as measures became stricter. Yet, this small decrease was brief, possibly because people started to notice positive aspects e.g., of spending more time at home, as the co-occurrence of words related to social distancing with terms like home, books, team, family, hug etc. might suggest. These findings suggest at least a short-term resilience to the challenges during the early phase of the COVID-19 outbreak.

Our results demonstrate that emotions during a long-term threat of a pandemic follow a different time course than after one-off events. In particular, they reveal some of the longest time periods of sustained emotional expression on social media observed so far. Multiple and repeated events can elicit emotions during a pandemic. Yet, without social interaction, individual emotional expressions on social media usually quickly relax back to the baseline (Pellert, Schweighofer, et al., 2020). In the absence of sufficiently strong social interaction, repeated pandemic events would produce emotional expression timelines with brief jumps that quickly vanish. In contrast, interaction between people can continuously reactivate emotions, making duration one potential indicator for collective emotions (Rimé ,2009; Goldenberg et al., 2020). Given that all people were personally concerned by the crisis, and strong emotions motivate social sharing (Rimé et al., 2009), such collective dynamics likely contributed to the enduring changes. Second, unlike previous studies of social media emotions after other catastrophic events (e.g. Garcia \& Rimé, 2019), we observed differences in the direction and timing of changes in negative emotional expressions: anxiety increased first and sadness later, whereas anger decreased. In this pandemic context, anxiety expression seems associated with cases and the onset of measures, and relaxes gradually once measures have been implemented. People seem to habituate to the new circumstances, or feel reassured by their governments' actions. Anger expressions drop as discourse on social media shifts away from politically polarized discussions. Finally, sadness expression seems mostly impacted by measures on people's personal lives, and only occurs in tweets mentioning COVID-19 deaths as these become more prevalent.

One of the most noticeable features of our results is their robustness across countries for negative emotions. All countries showed increases in anxiety terms, almost all in sadness terms, and most a decrease in anger expressions. Nonetheless, there was between-country 
variation, most clearly visible in baseline levels (i.e. random intercepts in models), but also in the size and timing of emotional changes. Differences in the size of the change in anxiety expression, as well as the particularly frequent terms in tweets, seem partly related to the number of cases. We observed a moderately strong positive association of anxiety terms and cases in both week 1 and 5 after the outbreak, as well as across the five weeks on average. Stronger correlations in week 1 and 5 compared to other weeks might reflect the first noticeable and the strongest absolute increase in nationwide cases per capita, respectively. Anxiety expression first rose in countries with quick and early case growth (Ireland, New Zealand, Switzerland, Austria, Figure S5). The highest peak occurred in Italy, during the severe crisis in Lombardia, which was frequently mentioned in anxiety terms (Figure S2.8). In the following weeks, anxiety expression seems less related to case growth. Five weeks after the outbreak, anxiety terms had decreased in most countries, but remained stable in some countries with larger case growth (Switzerland, Belgium, Italy, Austria, Ireland).

Latin American countries, which were more affected toward the end of the time period analyzed, nevertheless all implemented very strict measures quite quickly after the outbreak (see Figure S6 in Ecuador, Peru, Mexico, Chile). This may partially explain why anxiety terms did not increase as much as in other countries, which were hit when people may have felt less prepared. Additionally, protests for social justice and against violence against women in Chile and Mexico may have delayed attention to the pandemic. Interestingly, some of these countries experienced a strong growth in cases later (Chile and Peru in May/June, Ecuador in late April), which may hint at the function of anxiety in anticipating and reacting to danger. The case of Spain shows some similarities: The pattern of emotions, including only moderate frequency of anxiety terms but an increase in anger expressions, may reflect the politically polarized context in Spain, which possibly contributed to the severe outbreak observed afterwards. Frequent mentions of terms like government, state and measures, going out and protection in anxiety and/or anger tweets are consistent with this. The US, finally, shows smaller changes than all other English speaking countries for all four emotions, potentially because averaging across federal states reduced variance. Future studies focusing on the US could address this limitation (see also Ashokkumar \& Pennebaker, 2021; Valdez et al, 2020). Regarding other potential drivers of country-differences (beyond cases and measures), we noticed potentially languagerelated differences. For instance, Dutch, Spanish, Italian, and French speaking countries show smaller changes in anger expressions than most English and German speaking countries. In addition, cultural values and beliefs about emotions could explain variation between countries (Hsu et al., 2021).

Future research should explore the further trajectories of the investigated emotions: preliminary results of social media analyses for single countries (US: Saha et al., 2020, Ashwini et al. 2020,) suggest that, around May 2020, anxiety and sadness terms faded away, e.g. in Austria (Pellert, Lasser et al., 2020), or stabilized at a lower level, e.g. in the US (Ashokkumar et al., 2020). This may indicate that people got used to living under the new circumstances. Anger expression remained below the baseline in both of these countries. It remains unclear how the investigated emotions developed in response to further waves of the pandemic, or as social and economic consequences magnified, and as acceptance of measures may have changed. One limitation of our study is that positive emotions are measured as one broad category, given that some available validated and automated text analysis methods cannot distinguish between distinct 
positive emotions (e.g. Barbieri et al., 2020, Mohammad, 2021). Future research that manages to illustrate the diversity of positive emotional responses to crises not only with surveys (see e.g. Sun, Balabanova et al. 2020), but also social media data, would be very valuable.

Large-scale historical and real-time measurements of emotional expression on social media allows capturing collective emotional responses at a high time resolution across many countries and languages, including a baseline period before the event of interest. Although surveys lack this temporal resolution for large-scale emergent phenomena like COVID-19, and are rarely implemented across many countries, they can generate representative samples and more directly assess emotions via self-reports. Given that Twitter users are younger, more liberal, have higher formal education than the general population, and include more men (see e.g. Gallotti et al., 2020), future research should therefore assess the generalizability of our findings to larger populations (Sen et al., 2019). Nevertheless, the widespread penetration of social media across countries allows reaching populations traditionally underrepresented in psychological studies, for example low and middle income countries and non-English speakers.

When interpreting our results, one should further keep in mind that indirect measures of emotion via emotional expressions in text cannot distinguish between actual feelings and mere conversations about emotions. In addition to current and recent emotional states, emotional expressions may reflect other people's emotions, or cultural values about emotions (Hsu et al., 2021). For instance, users might post excitement when they believe they should be excited. This issue needs consideration in the interpretation of our results, especially when comparing between different countries. Nonetheless, when investigating collective emotions in large groups of people, discussions about others and social expectations are a crucial part of the target phenomenon. They may even provide information about people not active on the platform (Galesic et al., 2021; Garcia et al., 2021). Additionally, the indirect text-analysis measures of emotion do not suffer from recollection or self-reporting biases, which are problematic in survey research. Overall, social media analyses are thus a valuable complementary approach in addition to surveys for the investigation of collective emotions, but should not be considered a replacement.

Although we attempted to identify factors that may have influenced emotional responses by comparing countries, between-country differences need to be interpreted with caution. First, although we included tweets from more countries than previous studies, our sample is limited to 18 countries and correlations may be biased by outliers. Second, language differences also manifest in the LIWC dictionaries we used, which e.g. include different numbers of words in different languages. Some large between-language differences in the average level of tweets with emotion terms highlight the need for caution in interpreting cross-language comparisons (see Mohammad, 2021). Average levels were much more similar in countries with the same native language (Table S1). Finally, many other not yet mentioned factors might explain variation in emotions (media attention to events, political climate, etc).

A further limitation of our study is that the country-level aggregates of emotions might have missed emotional changes in smaller regions that were pandemic hot spots, and overestimate them in other less concerned regions. This particularly applies to large countries, and those implementing regulations at a federal state level (e.g. Germany, Italy, US). Nevertheless, our separate analysis of emotions for California and New York suggests that the key changes in 
anxiety and sadness expressions are comparable to the US aggregate time series. Finally, given the overall increase in tweets and active users during COVID-19, our results may not only capture changes in the emotional state of Twitter users from before to after the outbreak, but also reflect a change in the sample of Twitter users and general Twitter usage. Yet, a change in people's emotional state related to events during the outbreak may also be part of the reasons why some users became active on Twitter. The reported emotional changes should therefore be interpreted at the population level rather than a within-subject change in Twitter users.

From a risk communication standpoint, the role of a timely understanding of and response to emotional states in the public as they occur and change during a crisis has been increasingly acknowledged in recent years. As the primary goal of risk communication during an outbreak (or other public emergency) is to communicate in ways that build, maintain or restore public trust, the emotional state of the public is highly relevant to connect to the audience and maintain that connection over time (Heymann, 2008). Behavioral research indicates that the general public becomes more concerned the less it trusts in the communicators (Heymann, 2008), which is, in turn, relevant to their reaching of conclusions, e.g. about their willingness to comply with recommendations (Buck \& Ferrer, 2012). Therefore, adequately addressing emotional states is essential to enhance trust, and can inform the construction of effective warnings which need to resonate with the public audience (Turner, 2007). By providing timely and easily accessible indicators of a population's collective emotional state, social media analyses like this one could help to improve official risk and emergency communication.

Acknowledgements: This work was supported by two grants from the Vienna Science and Technology Fund with the Grant No. VRG16-005 and COV20-027 to D. Garcia, H. Metzler, A. Di Natale and T. Niederkrotenthaler. Access to the Crimson Hexagon database was provided via the project V!brant Emotional Health grant "Suicide Prevention media campaign Oregon" to T. Niederkrotenthaler.

\section{References}

Agosti, A., \& Rellini, A. (2007). The Italian LIWC dictionary. Austrian, TX: Technical report, LIWC. net.

American Psychiatric Association (2013). Diagnostic and statistical manual of mental disorders (5th ed.). Author. https://doi.org/10.1176/appi.books.9780890425596

Ashokkumar, A., \& Pennebaker, J. W. (2021). Social media conversations reveal large psychological shifts caused by COVID-19's onset across U.S. cities. Science Advances, 7(39), eabg7843. https://doi.org/10.1126/sciadv.abg7843

Auguie, B. (2017). gridExtra: Miscellaneous Functions for "Grid" Graphics. https://CRAN.Rproject.org/package=gridExtra

Barbieri, F., Camacho-Collados, J., Neves, L., \& Espinosa-Anke, L. (2020). TweetEval: Unified Benchmark and Comparative Evaluation for Tweet Classification. ArXiv:2010.12421 [Cs]. http://arxiv.org/abs/2010.12421

Bates, D., Mächler, M., Bolker, B., \& Walker, S. (2015). Fitting linear mixed-effects models using Ime4. Journal of Statistical Software, 67(1). https://doi.org/10.18637/jss.v067.i01

Boot, P., Zijlstra, H., \& Geenen, R. (2017). The Dutch translation of the Linguistic Inquiry and 
Word Count (LIWC) 2007 dictionary. Dutch Journal of Applied Linguistics, 6(1), 65-76. https://doi.org/10.1075/dujal.6.1.04boo

Buck, R., \& Ferrer, R. (2012). Emotion, Warnings, and the Ethics of Risk Communication. In S. Roeser, R. Hillerbrand, P. Sandin, \& M. Peterson (Eds.), Handbook of Risk Theory: Epistemology, Decision Theory, Ethics, and Social Implications of Risk (pp. 693-723). Springer Netherlands. https://doi.org/10.1007/978-94-007-1433-5_27

Bults, M., Beaujean, D. J., de Zwart, O., Kok, G., van Empelen, P., van Steenbergen, J. E., Richardus, J. H., \& Voeten, H. A. (2011). Perceived risk, anxiety, and behavioural responses of the general public during the early phase of the Influenza $A(H 1 N 1)$ pandemic in the Netherlands: Results of three consecutive online surveys. BMC Public Health, 11(1), 2. https://doi.org/10.1186/1471-2458-11-2

Castaldo, M., Venturini, T., Frasca, P., \& Gargiulo, F. (2021). The rhythms of the night: Increase in online night activity and emotional resilience during the spring 2020 Covid-19 lockdown. EPJ Data Science, 10(1), 1-15. https://doi.org/10.1140/epjds/s13688-021-00262-1

Cha, M., Gwon, Y., \& Kung, H. (2015). Twitter Geolocation and Regional Classification via Sparse Coding. Proceedings of the International AAAI Conference on Web and Social Media, 9(1), Article 1. https://ojs.aaai.org/index.php/ICWSM/article/view/14664

Coombs, W. T. (2007). Protecting Organization Reputations During a Crisis: The Development and Application of Situational Crisis Communication Theory. Corporate Reputation Review, 10(3), 163-176. https://doi.org/10.1057/palgrave.crr.1550049

Desvars-Larrive, A., Dervic, E., Haug, N., Niederkrotenthaler, T., Chen, J., Di Natale, A., Lasser, J., Gliga, D. S., Roux, A., Sorger, J., Chakraborty, A., Ten, A., Dervic, A., Pacheco, A., Jurczak, A., Cserjan, D., Lederhilger, D., Bulska, D., Berishaj, D., ... Thurner, S. (2020). A structured open dataset of government interventions in response to COVID-19. Scientific Data, 7(1), 285. https://doi.org/10.1038/s41597-020-00609-9

Dong, E., Du, H., \& Gardner, L. (2020). An interactive web-based dashboard to track COVID-19 in real time. The Lancet Infectious Diseases, 20(5), 533-534. https://doi.org/10.1016/S1473-3099(20)30120-1

Doré, B., Ort, L., Braverman, O., \& Ochsner, K. N. (2015). Sadness Shifts to Anxiety Over Time and Distance From the National Tragedy in Newtown, Connecticut. Psychological Science, 26(4), 363-373. https://doi.org/10.1177/0956797614562218

Durkheim, É. (1915). The elementary forms of religious life (J. D. Swan transl.). Georges Allen \& Unwin Ltd (Original work published 1912).

Fredrickson, B. L., Tugade, M. M., Waugh, C. E., \& Larkin, G. R. (2003). What Good Are Positive Emotions in Crises? A Prospective Study of Resilience and Emotions Following the Terrorist Attacks on the United States on September 11th, 2001. Journal of Personality and Social Psychology, 84(2), 365-376.

Frijda, N. H. (1986). The emotions. Cambridge University Press.

Galesic, M., Bruine de Bruin, W., Dalege, J., Feld, S. L., Kreuter, F., Olsson, H., Prelec, D., Stein, D. L., \& van der Does, T. (2021). Human social sensing is an untapped resource for computational social science. Nature, 1-9. https://doi.org/10.1038/s41586-021-03649-2

Gallotti, R., Valle, F., Castaldo, N., Sacco, P., \& De Domenico, M. (2020). Assessing the risks of 'infodemics' in response to COVID-19 epidemics. Nature Human Behaviour, 4(12), 12851293. https://doi.org/10.1038/s41562-020-00994-6

Garcia, D., Pellert, M., Lasser, J., \& Metzler, H. (2021). Social media emotion macroscopes reflect emotional experiences in society at large. ArXiv:2107.13236 [Cs]. http://arxiv.org/abs/2107.13236

Garcia, D., \& Rimé, B. (2019). Collective Emotions and Social Resilience in the Digital Traces After a Terrorist Attack. Psychological Science, 30(4), 617-628. https://doi.org/10.1177/0956797619831964

Goldenberg, A., Garcia, D., Halperin, E., \& Gross, J. J. (2020). Collective Emotions. Current 
Directions in Psychological Science, 29(2), 154-160.

https://doi.org/10.1177/0963721420901574

Gruebner, O., Lowe, S. R., Sykora, M., Shankardass, K., Subramanian, S. V., \& Galea, S. (2017). A novel surveillance approach for disaster mental health. PLOS ONE, 12(7), e0181233. https://doi.org/10.1371/journal.pone.0181233

Hale, T., Angrist, N., Goldszmidt, R., Kira, B., Petherick, A., Phillips, T., Webster, S., CameronBlake, E., Hallas, L., Majumdar, S., \& Tatlow, H. (2021). A global panel database of pandemic policies (Oxford COVID-19 Government Response Tracker). Nature Human Behaviour, 5(4), 529-538. https://doi.org/10.1038/s41562-021-01079-8

Heymann, D. L. (Ed.). (2008). Control of Communicable Diseases Manual. American Public Health Association. https://doi.org/10.2105/CCDM.2745

Holman, E. A., Thompson, R. R., Garfin, D. R., \& Silver, R. C. (2020). The unfolding COVID-19 pandemic: A probability-based, nationally representative study of mental health in the United States. Science Advances, 6(42), eabd5390. https://doi.org/10.1126/sciadv.abd5390

Hsu, T. W., Niiya, Y., Thelwall, M., Ko, M., Knutson, B., \& Tsai, J. L. (2021). Social media users produce more affect that supports cultural values, but are more influenced by affect that violates cultural values. Journal of Personality and Social Psychology, 121(5), 969-983. https://doi.org/10.1037/pspa0000282

Jaidka, K., Giorgi, S., Schwartz, H. A., Kern, M. L., Ungar, L. H., \& Eichstaedt, J. C. (2020). Estimating geographic subjective well-being from Twitter: A comparison of dictionary and data-driven language methods. Proceedings of the National Academy of Sciences. https://doi.org/10.1073/pnas. 1906364117

Jones, J. H., \& Salathé, M. (2009). Early Assessment of Anxiety and Behavioral Response to Novel Swine-Origin Influenza A(H1N1). PLOS ONE, 4(12), e8032. https://doi.org/10.1371/journal.pone.0008032

Jones, N. M., \& Silver, R. C. (2020). This is not a drill: Anxiety on Twitter following the 2018 Hawaii false missile alert. The American Psychologist, 75(5), 683-693. https://doi.org/10.1037/amp0000495

Jones, N. M., Thompson, R. R., Schetter, C. D., \& Silver, R. C. (2017). Distress and rumor exposure on social media during a campus lockdown. Proceedings of the National Academy of Sciences, 114(44), 11663-11f668. https://doi.org/10.1073/pnas.1708518114

Jones, N. M., Wojcik, S. P., Sweeting, J., \& Silver, R. C. (2016). Tweeting negative emotion: An investigation of Twitter data in the aftermath of violence on college campuses.

Psychological Methods, 21(4), 526-541. https://doi.org/10.1037/met0000099

Jungmann, S. M., \& Witthöft, M. (2020). Health anxiety, cyberchondria, and coping in the current COVID-19 pandemic: Which factors are related to coronavirus anxiety? Journal of Anxiety Disorders, 73, 102239. https://doi.org/10.1016/j.janxdis.2020.102239

Karademas, E. C., Bati, A., Karkania, V., Georgiou, V., \& Sofokleous, S. (2013). The association between Pandemic Influenza A (H1N1) public perceptions and reactions: A prospective study. Journal of Health Psychology, 18(3), 419-428. https://doi.org/10.1177/1359105312436765

Kim, H. K., \& Niederdeppe, J. (2013). The Role of Emotional Response during an H1N1 Influenza Pandemic on a College Campus. Journal of Public Relations Research, 25(1), 30-50. https://doi.org/10.1080/1062726X.2013.739100

Lerner, J. S., \& Keltner, D. (2001). Fear, anger, and risk. Journal of Personality and Social Psychology, 81(1), 146-159. https://doi.org/10.1037//0022-3514.81.1.146

Liu, Y., Ott, M., Goyal, N., Du, J., Joshi, M., Chen, D., Levy, O., Lewis, M., Zettlemoyer, L., \& Stoyanov, V. (2019). RoBERTa: A Robustly Optimized BERT Pretraining Approach. ArXiv:1907.11692 [Cs]. http://arxiv.org/abs/1907.11692

Lwin, M. O., Lu, J., Sheldenkar, A., Schulz, P. J., Shin, W., Gupta, R., \& Yang, Y. (2020). Global 
Sentiments Surrounding the COVID-19 Pandemic on Twitter: Analysis of Twitter Trends. JMIR Public Health and Surveillance, 6(2), e19447. https://doi.org/10.2196/19447

Meadows, C. W., Meadows, C. Z., Tang, L., \& Liu, W. (2019). Unraveling Public Health Crises Across Stages: Understanding Twitter Emotions and Message Types During the California Measles Outbreak. Communication Studies, 70(4), 453-469. https://doi.org/10.1080/10510974.2019.1582546

Metzler, H., Rimé, B., Pellert, M., Niederkrotenthaler, T., Di Natale, A., \& Garcia, D. (2021, June 24). Collective Emotions during the Covid-19 Outbreak. Retrieved from osf.io/736kc

Mohammad, S., Bravo-Marquez, F., Salameh, M., \& Kiritchenko, S. (2018). SemEval-2018 Task 1: Affect in Tweets. Proceedings of The 12th International Workshop on Semantic Evaluation, 1-17. https://doi.org/10.18653/v1/S18-1001

Mohammad, S. M. (2021). Chapter 11 - Sentiment analysis: Automatically detecting valence, emotions, and other affectual states from text. In H. L. Meiselman (Ed.), Emotion Measurement (Second Edition) (pp. 323-379). Woodhead Publishing. https://doi.org/10.1016/B978-0-12-821124-3.00011-9

Oatley, K., \& Johnson-Laird, P. N. (2014). Cognitive approaches to emotions. Trends in Cognitive Sciences, 18(3), 134-140. https://doi.org/10.1016/j.tics.2013.12.004

Oscar, N., Fox, P. A., Croucher, R., Wernick, R., Keune, J., \& Hooker, K. (2017). Machine Learning, Sentiment Analysis, and Tweets: An Examination of Alzheimer's Disease Stigma on Twitter. The Journals of Gerontology: Series B, 72(5), 742-751.

https://doi.org/10.1093/geronb/gbx014

Pagnini, F., Bonanomi, A., Tagliabue, S., Balconi, M., Bertolotti, M., ... Villani, D. (2020). Knowledge, Concerns, and Behaviors of Individuals During the First Week of the Coronavirus Disease 2019 Pandemic in Italy. JAMA Network Open, 3(7), e2015821. https://doi.org/10.1001/jamanetworkopen.2020.15821

Peitz, L., Lalot, F., Douglas, K., Sutton, R., \& Abrams, D. (2021). COVID-19 conspiracy theories and compliance with governmental restrictions: The mediating roles of anger, anxiety, and hope. Journal of Pacific Rim Psychology, 15, 18344909211046650. https://doi.org/10.1177/18344909211046646

Pellert, M., Lasser, J., Metzler, H., \& Garcia, D. (2020). Dashboard of sentiment in Austrian social media during COVID-19. Frontiers in Big Data, 3. https://doi.org/10.3389/fdata.2020.00032

Pellert, M., Metzler, H., Matzenberger, M., \& Garcia, D. (2021). Validating daily social media macroscopes of emotions. ArXiv:2108.07646 [Cs]. http://arxiv.org/abs/2108.07646

Pellert, M., Schweighofer, S., \& Garcia, D. (2020). The individual dynamics of affective expression on social media. EPJ Data Science, 9(1), 1-14. https://doi.org/10.1140/epjds/s13688-019-0219-3

Pennebaker, J. W., Boyd, R. L., Jordan, K., \& Blackburn, K. (2015). The Development and Psychometric Properties of LIWC2015. https://repositories.lib.utexas.edu/handle/2152/31333

Pennebaker, J. W., \& Harber, K. D. (1993). A Social Stage Model of Collective Coping: The Loma Prieta Earthquake and The Persian Gulf War. Journal of Social Issues, 49(4), 125145. https://doi.org/10.1111/j.1540-4560.1993.tb01184.x

Piolat, A., Booth, R. J., Chung, C. K., Davids, M., \& Pennebaker, J. W. (2011). La version française du dictionnaire pour le LIWC: Modalités de construction et exemples d'utilisation. Psychologie Française, 56(3), 145-159. https://doi.org/10.1016/j.psfr.2011.07.002

$\mathrm{R}$ Core Team. (2018). R: A language and environment for statistical computing. R Foundation for Statistical Computing. https://www.R-project.org/

Ramírez-Esparza, N., Pennebaker, J. W., García, F. A., \& Suriá Martínez, R. (2007). La psicología del uso de las palabras: Un programa de computadora que analiza textos en español. http://rua.ua.es/dspace/handle/10045/25918 
Rimé, B. (2009). Emotion Elicits the Social Sharing of Emotion: Theory and Empirical Review. Emotion Review, 1(1), 60-85. https://doi.org/10.1177/1754073908097189

Rubin, G. J., Amlôt, R., Page, L., \& Wessely, S. (2009). Public perceptions, anxiety, and behaviour change in relation to the swine flu outbreak: Cross sectional telephone survey. BMJ, 339. https://doi.org/10.1136/bmj.b2651

Russell, J. A. (2003). Core affect and the psychological construction of emotion. Psychological Review, 110(1), 145-172. https://doi.org/10.1037/0033-295X.110.1.145

Saha, K., Torous, J., Caine, E. D., \& De Choudhury, M. (2020). Psychosocial Effects of the COVID-19 Pandemic: Large-scale Quasi-Experimental Study on Social Media. Journal of Medical Internet Research, 22(11), e22600 10.2196/22600

Sander, D. E., \& Scherer, K. R. (Eds.) (2009). The Oxford companion to emotion and the affective sciences. Oxford University Press.

Scherer, K. R. (1997). The role of culture in emotion-antecedent appraisal. Journal of Personality and Social Psychology, 73(5), 902-922. https://doi.org/10.1037/00223514.73.5.902

Sen, I., Floeck, F., Weller, K., Weiss, B., \& Wagner, C. (2019). A Total Error Framework for Digital Traces of Humans. ArXiv:1907.08228 [Cs]. http://arxiv.org/abs/1907.08228

Slowikowski, K. (2019). Ggrepel: Automatically Position Non-Overlapping Text Labels with "ggplot2". R package version 0.8.1. https://CRAN.R-project.org/package=ggrepel

Sun, R., Balabanova, A., Bajada, C. J., Liu, Y., Kriuchok, M., Voolma, ... Sauter, D. (2020). Psychological wellbeing during the global COVID-19 outbreak [Preprint]. PsyArXiv. https://doi.org/10.31234/osf.io/r7xaz

Sun, J., Schwartz, H. A., Son, Y., Kern, M. L., \& Vazire, S. (2020). The language of well-being: Tracking fluctuations in emotion experience through everyday speech. Journal of Personality and Social Psychology, 118(2), 364-387. https://doi.org/10.1037/pspp0000244

Turner, M. M. (2007). Using emotion in risk communication: The Anger Activism Model. Public Relations Review, 33(2), 114-119. https://doi.org/10.1016/j.pubrev.2006.11.013

Valdez, D., Thij, M. ten, Bathina, K., Rutter, L. A., \& Bollen, J. (2020). Social Media Insights Into US Mental Health During the COVID-19 Pandemic: Longitudinal Analysis of Twitter Data. Journal of Medical Internet Research, 22(12), e21418. https://doi.org/10.2196/21418

Wickham, H. et al. (2019). Welcome to the Tidyverse. Journal of Open Source Software, 4(43), 1686. https://doi.org/10.21105/joss.01686

Wickham, H., \& Seidel, D. (2019). Scales: Scale Functions for Visualization. R package version 1.1.0. https://CRAN.R-project.org/package=scales

Wilke, C. O. (2019). Cowplot: Streamlined Plot Theme and Plot Annotations for "ggplot2". R package version 1.0.0. https://CRAN.R-project.org/package $=$ cowplot

Wolf, M., Horn, A. B., Mehl, M. R., Haug, S., Pennebaker, J. W., \& Kordy, H. (2008). Computergestützte quantitative Textanalyse-Äquivalenz und Robustheit der deutschen Version des Linguistic Inquiry and Word Count. Diagnostica, 54(2), 85-98. https://doi.org/10.1026/0012-1924.54.2.85

Yarrington, J. S., Lasser, J., Garcia, D., Vargas, J. H., Couto, D. D., Marafon, T., Craske, M. G., \& Niles, A. N. (2021). Impact of the COVID-19 Pandemic on Mental Health among 157,213 Americans. Journal of Affective Disorders, 286, 64-70. https://doi.org/10.1016/j.jad.2021.02.056 